\title{
RECENT PROGRESS OF LASER METROLOGY IN CHEMICALLY REACTING FLOWS AT ONERA
}

\author{
A. Mohamed, N. Dorval, G. Vilmart, M. Orain, \\ R. George, M. Scherman, M. Nafa, A. Bresson, \\ B. Attal-Tretout, and M. Lefebvre
}

ONERA

The French Aerospace Lab.

Centre de la Hunière

BP 80100, Palaiseau 91123, France

\begin{abstract}
This paper presents some of the development actions performed these last years at ONERA using laser spectroscopic techniques to probe chemically reacting flows. Techniques like laser absorption, laser induced fluorescence (LIF), and Raman scattering will be described with focus on present drawbacks as well as expectations from new laser technologies (Interband Cascade Lasers (ICL) diodes, Optical Parametrical Oscillators (OPO), frequency comb, and femto/picosecond lasers) before showing some results of recent applications in ground facilities.
\end{abstract}

\section{INTRODUCTION}

During these last decades, nonintrusive spectroscopic measurement techniques have been and are still being developed to probe chemically reacting flows as these tools are now well accepted to provide insights on internal states of atoms and molecules which impacts chemical reactions and nonequilibrium thermodynamic properties in the studied gases. For aerodynamic and combustion applications, ONERA has been developing and applying techniques like absorption spectroscopy with different laser sources (diode laser, OPO, frequency comb), LIF with emphasis on high repetition rate measurements or recent applications on atomic species catalyzers in combustion, Raman spectroscopy with pico/femtosecond lasers, and electron beam fluorescence with new miniature guns for inflight purposes.

(C) The authors, published by EDP Sciences. This is an Open Access article distributed under the terms of the Creative Commons Attribution License 4.0 (http://creativecommons.org/licenses/by/4.0/). 


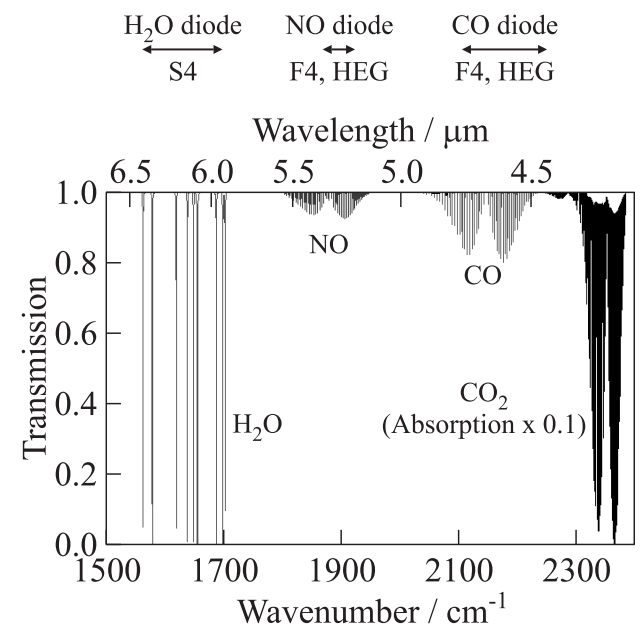

(a)

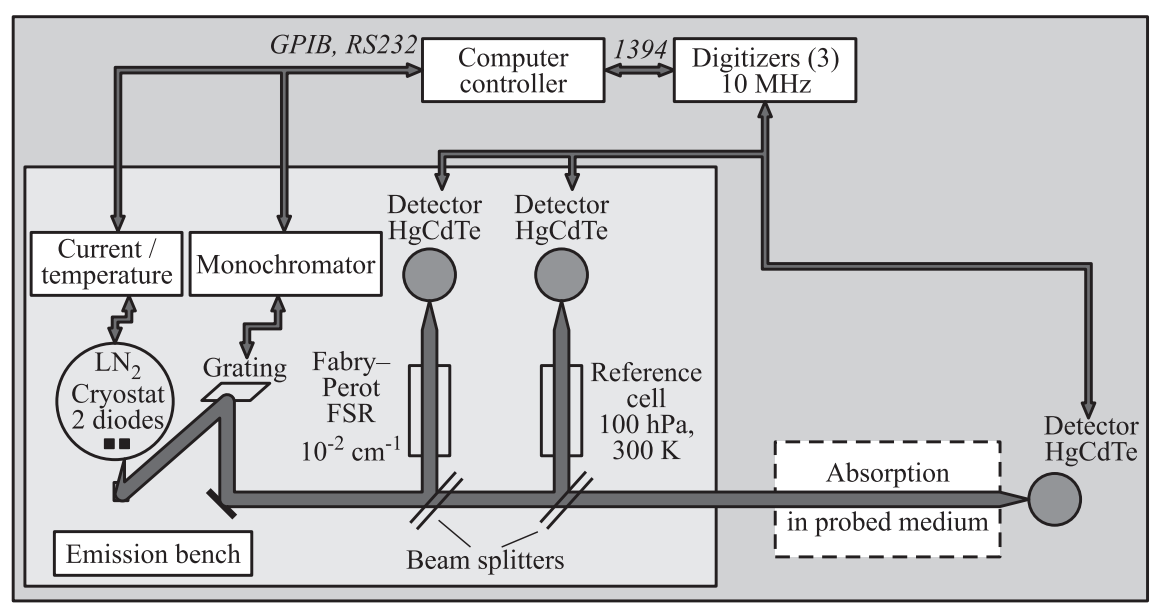

(b)

Figure 1 Typical absorption spectrum of some molecules of aerodynamic interest and spectral emission windows of some of the laser diodes used in wind tunnels and combustion applications [1] (HEG - High-Enthalpy shock tunnel in Göttingen) ( $a$ ) and generic TDLAS spectrometer (FSR - Free Spectral Range) (b) 


\section{LASER ABSORPTION SPECTROSCOPY}

Laser absorption spectroscopy remains one of the most robust spectroscopic techniques with which one can measure velocity, temperatures, and species concentrations [1] in many aerodynamic and combustion applications.

At ONERA, different setups based on different laser sources (diode lasers, $\mathrm{OPO}$, frequency comb, etc.) are continuously being developed and applied in its own facilities or for external customers.

\subsection{Tunable Diode Laser Absorption Spectroscopy}

Tunable diode laser absorption spectroscopy (TDLAS) technique has been much used to characterize high-enthalpy flows using cryogenic lead salt diode lasers which allow probing relevant molecules having their fundamental absorption lines in the mid-infrared spectral region (Fig. 1a).

Setups using these laser sources (Fig. 1b) are usually bulky and complicated to operate. Nowadays, development actions concern building of compact and transportable setups taking advantage of recently available noncryogenic midinfrared semiconductor lasers (ICL diodes and quantum cascade lasers (QCL)) or OPO.

Figures 2 and 3 present some typical spectra recorded for combustion or hypersonic flows as well as the parameters which can be determined from their interpretation keeping in mind that this technique is of line-of-sight nature giving only integrated values along the absorption path of the laser used.

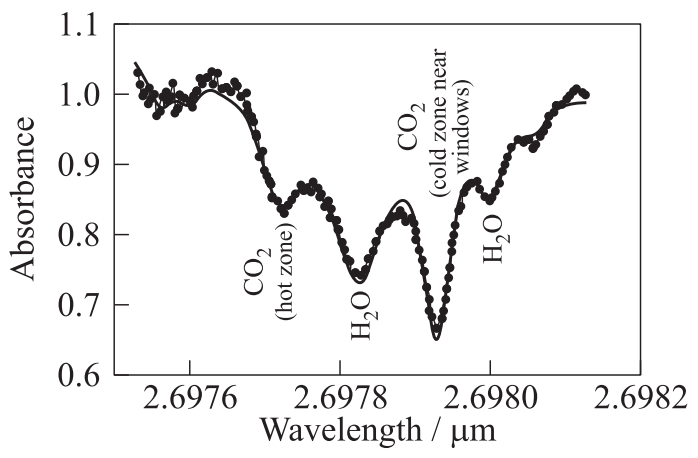

Figure 2 Typical absorption spectra in the combustion flows: $\mathrm{CO}_{2}$ density $3.6 \cdot 10^{17} \mathrm{~cm}^{-3} ; \mathrm{H}_{2} \mathrm{O}$ density $=3.4 \cdot 10^{17} \mathrm{~cm}^{-3} ; T=1530 \mathrm{~K}$; and uncertainties $= \pm 10 \%$. Signs refer to experiments and curves to best fit 


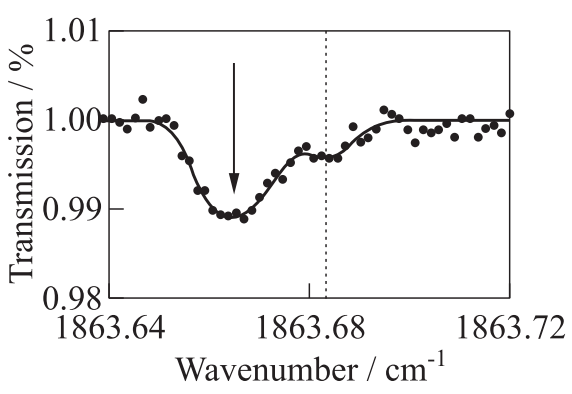

(a)

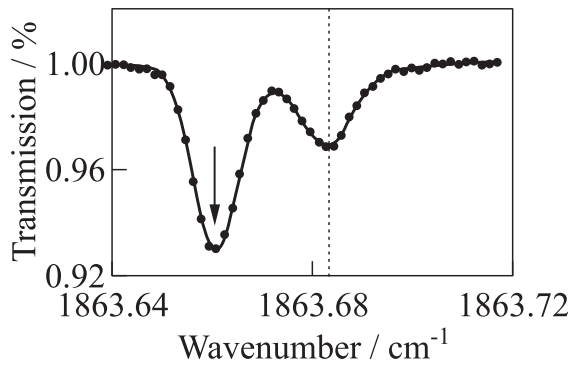

(c)

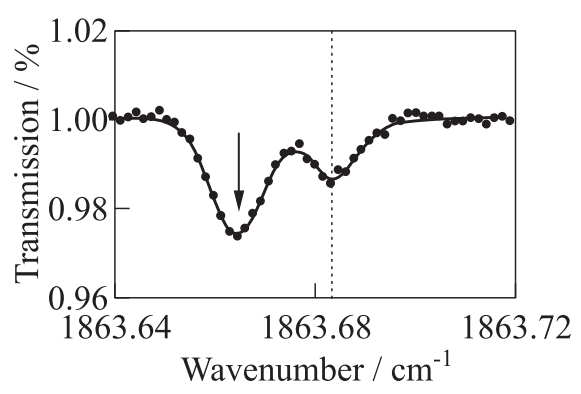

(b)

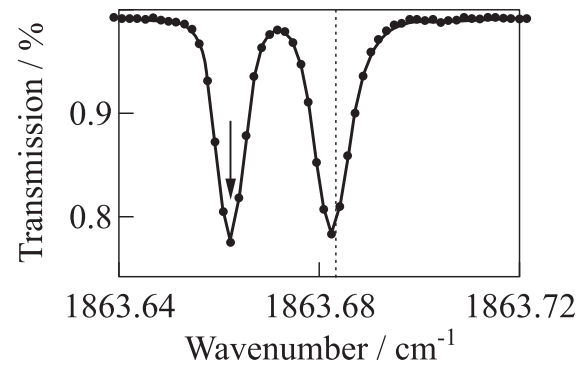

(d)

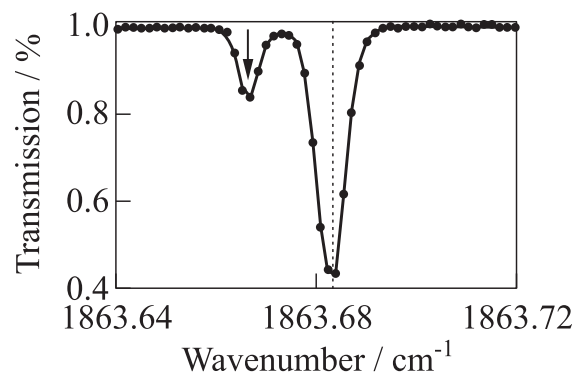

(e)

Figure 3 Typical absorption spectra in the hypersonic flows: $(a) t=0.1 \mathrm{~ms}, V$ $=6324 \mathrm{~m} / \mathrm{s}, T_{\text {Dop }}=1678 \mathrm{~K}$, and $T_{\text {rest }}=1495 \mathrm{~K} ;(b) t=0.5 \mathrm{~ms}, V=5316 \mathrm{~m} / \mathrm{s}$, $T_{\text {Dop }}=2376 \mathrm{~K}$, and $T_{\text {rest }}=1927 \mathrm{~K} ;(c) t=1 \mathrm{~ms}, V=5925 \mathrm{~m} / \mathrm{s}, T_{\text {Dop }}=1786 \mathrm{~K}$, and $T_{\text {rest }}=2051 \mathrm{~K} ;(d) t=3 \mathrm{~ms}, V=5500 \mathrm{~m} / \mathrm{s}, T_{\text {Dop }}=644 \mathrm{~K}$, and $T_{\text {rest }}=949 \mathrm{~K}$; and $(e) t=9 \mathrm{~ms}, V=3815 \mathrm{~m} / \mathrm{s}, T_{\text {Dop }}=857 \mathrm{~K}$, and $T_{\text {rest }}=682 \mathrm{~K}$. Signs refer to experiments and curves to best fit 


\subsection{Frequency Comb Fourier Spectroscopy}

Interband cascade lasers and other recently developed lasers have opened new possibilities in absorption spectroscopy but there are still many limitations with these light sources exploited up to now. For example, they seldom offer continuous spectral tunability in a window larger than $1 \mathrm{~cm}^{-1}$ limiting probing to only one or a few absorption lines. This can be sufficient for velocity or temperature measurements. But for species density or vibrational temperature measurements in high-enthalpy flows which are often in nonequilibrium, more lines are needed to sample properly the population distribution among the different energy levels of a specie. Additional lines from different species are also required to correctly interpret dissociation features. For example, in the case of a Martian reentry flow, to acquire simultaneous $\mathrm{CO}_{2}, \mathrm{CO}$, and NO lines, a broadband coverage of the spectral region between 1800 and $2400 \mathrm{~cm}^{-1}$ (i. e., a band of $600 \mathrm{~cm}^{-1}$, cf. Fig. 1) is needed. A high spectral resolution of about $10^{-3} \mathrm{~cm}^{-1}$ is simultaneous needed to discriminate without ambiguity the lines as well as to extract information like temperature or velocity from line profiles. Several diode lasers are needed leading to a complex laser excitation setup. The third simultaneous requirement is a short acquisition time with a high repetition rate (higher than $10 \mathrm{kHz}$ ) to follow adequately the time evolution of transient phenomena. Recent progress in laser development brings the possible solution of supercontinuum laser but up to now, light emission is limited in the near infrared (less than $2 \mu \mathrm{m}$ ) not covering the fundamental bands of the species mentioned above which are mostly situated at wavelengths superior to $4 \mu \mathrm{m}$. On the detection side, spectral dispersion of a very large spectral band is very difficult with classical means like a grating or a prism to observe the absorption spectra with adequate spectral resolution.

One of the most promising solutions seems to be in frequency comb spectroscopy, particularly, a setup using optical beating between two combs leading to a Fourier-type broadband spectroscopy with high spectral resolution [2] and spectra acquisition repetition rates which have the potential to approach the megahertz regime. However, as for supercontinuum lasers, actual off-theshelf technology provides mostly combs in the visible or near-infrared spectral regions [3]. But research nowadays with different sources and methods (difference frequency generation (DFG) conversion [2], Fabry-Perot frequency comb QCL, microresonators, etc.) presents excellent prospects to have soon frequency combs in the mid-infrared. A frequency comb laser can be distinguished by its spectrum consisting of a series of discrete and equally spaced peaks (or teeth) which can be stable and accurate at the subhertz level and which can span over more than one octave with a few millions peaks [4]. Lasers exhibiting such emissions obtained using a particular mode locking mechanism became a reality from the development Ti:sapphire femtosecond lasers in the 1990's and from the pioneering works of John L. Hall [5] and Theodor W. Hänsch [6] in laser-based 
precision frequency metrology [6] leading to their winning of the Nobel Prize in 2005 .

Absorption spectroscopy using a single frequency comb has already been demonstrated in several applications. As mentioned above, one of the major difficulties is within the detection scheme which does not allow exploiting all the potential of the high spectral resolution coupled to a broad spectral emission window. One can restrict measurements into a small portion of the spectral window. Another solution can be in the use of a special two-dimensional grating like the VIPA (Virtually Imaged Phased Array) [7-9] coupled to a high-speed camera in the adequate spectral range. But this detection scheme and data processing are complex and yet to be demonstrated at other wavelengths than for the telecom range.

A more elegant solution lies in the new method exploiting the optical beating of two combs with slightly different repetition rates [2]. Although more costly (requiring a pair of lasers), it allows to benefit fully of the three advantages of frequency combs (broad spectral coverage, high spectral resolution, and high repetition rate). The optical beating between such two combs at repetition rates $f_{r}$ and $f_{r}+\Delta$, where $f_{r}$ is in the range $100 \mathrm{MHz}$ and $\Delta$ of a few tens of hertz, will provide different groups of waves at frequencies resulting from the mixing (sum and difference) of the peaks between the two combs. Usually, one focus on the particular group (zero order) having frequencies below $f_{r}$ which can easily be acquired and processed with radio-frequency components. These radio-frequencies can be expressed as

$$
f_{\mathrm{RF}}=n \Delta+f_{01}-f_{02}
$$

where $n$ is the integer and noting $f_{01}$ and $f_{02}$ as the offset frequencies of the two lasers. The accumulation of such waves on a simple photodectector delivers a signal which is the Fourier transform of the product of the combs. This method, now known as dual comb spectroscopy, was initiated by Shiller [10] in 2002 before full demonstrations by authors of $[2,11,12]$.

The described laser platform setup consists of two nearly identical sets of lasers, each set having a frequency comb generated from an Erbium fiber laser around $1.5 \mu \mathrm{m}$ at low power and which seeds two Erbium amplifiers, one to produce a comb of higher average power $(300 \mathrm{~mW})$ and the other to generate a supercontinuum emission between 1- and 2-micrometer wavelengths. Figure $4 a$ shows the emission envelope intensity (acquired with an ANDO AQ6317B turning monochromator) of one of the supercontinuum lasers together with indication of the positions of some weak absorption lines (or overtones) from species of interest for aerodynamic and combustion applications. The modulation of this envelope, which must not be confused to noise, is the result of the gain efficiencies of the different nonlinear phenomena (four wave mixing, self-phase modulation, etc.) occurring in the nonlinear fiber generating the supercontinuum emission. Figure $4 b$ presents a zoom of this supercontinuum spectrum at 


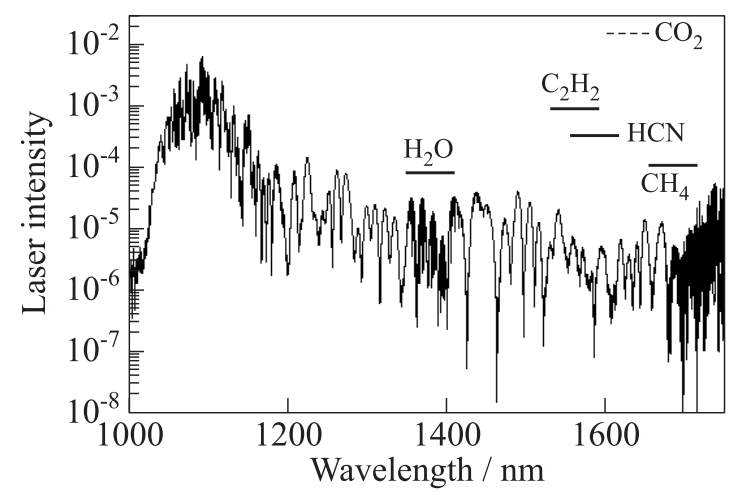

(a)
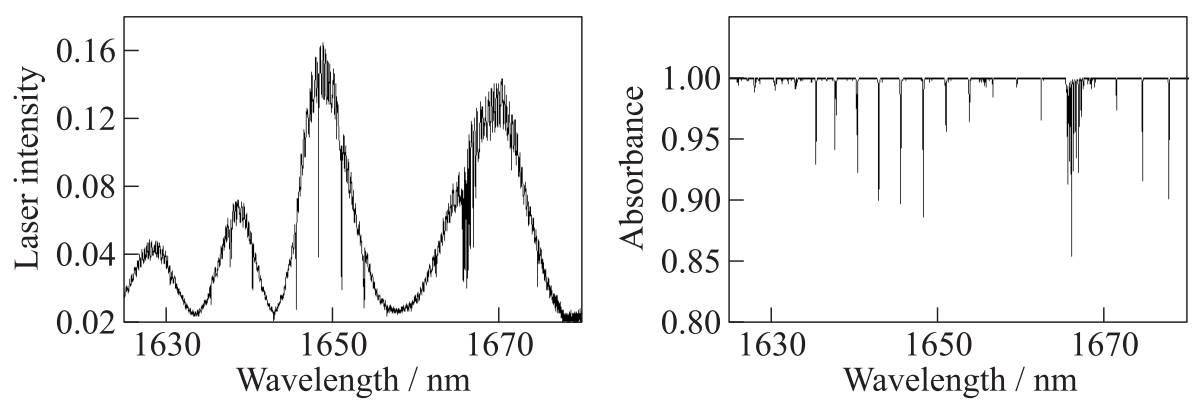

(b)

Figure 4 Supercontinuum envelope emission: ( $a$ ) whole range (mean power $300 \mathrm{~mW}$ ); and $(b)$ part of the supercontinuum output exhibiting absorption lines of $\mathrm{CH}_{4}$ (pressure 300 mbar and temperature $300 \mathrm{~K}$ )

the location of absorption lines of $\mathrm{CH}_{4}$. These lines correspond to the case when the laser crosses a 10-centimeter long gas cell filled with this specie at a pressure of 300 mbar at the temperature of $300 \mathrm{~K}$.

The envelope emission from one of the two frequency comb lasers is shown in Fig. $5 a$. The spectral coverage is limited to about $170 \mathrm{~nm}$ (from 1480 to $1650 \mathrm{~nm}$ ) and allows observations of only lines from $\mathrm{C}_{2} \mathrm{H}_{2}, \mathrm{HCN}$, and $\mathrm{CH}_{4}$. Figure $5 b$ shows the absorption spectrum of $\mathrm{C}_{2} \mathrm{H}_{2}$ obtained with this frequency comb laser in a 10-centimeter gas cell filled with this specie (300 mbar at $300 \mathrm{~K})$.

Figure $6 a$ presents an interferogram of dual comb Fourier spectroscopy applied to the $\mathrm{C}_{2} \mathrm{H}_{2}$ specie in a 10-centimeter long cell. The interferogram of the optical beating between the two combs is acquired at 10-kilohertz repetition rate with the help of a high-speed detector having a bandwidth over $200 \mathrm{MHz}$. The inverse Fourier transformation of this interferogram leads to the absorption 

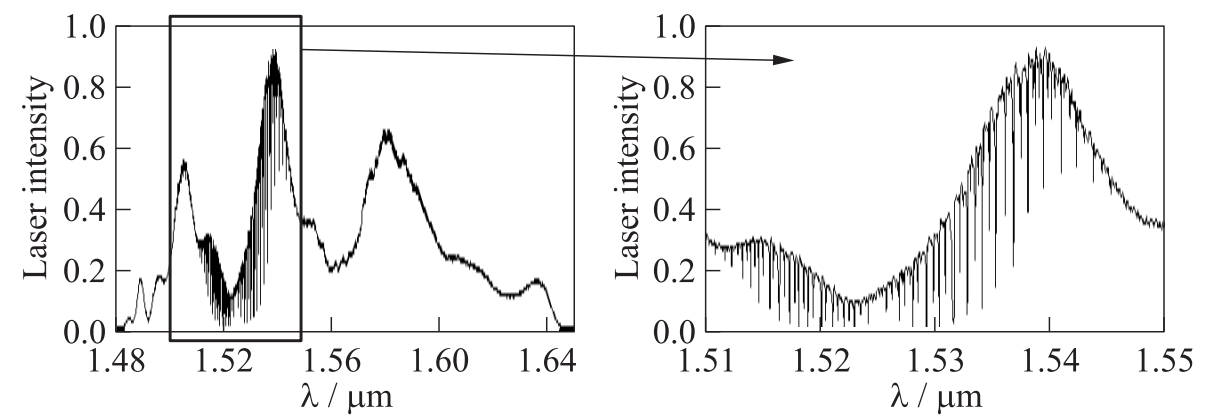

(a)

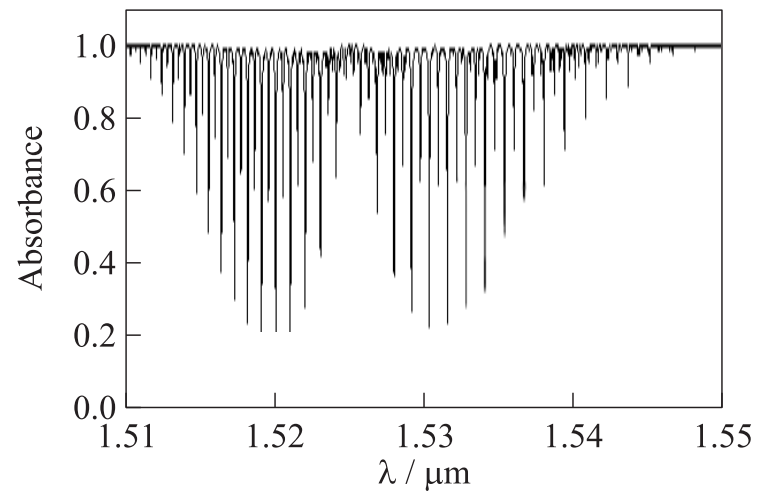

(b)

Figure 5 Part of the frequency comb output exhibiting absorption lines of $\mathrm{C}_{2} \mathrm{H}_{2}$

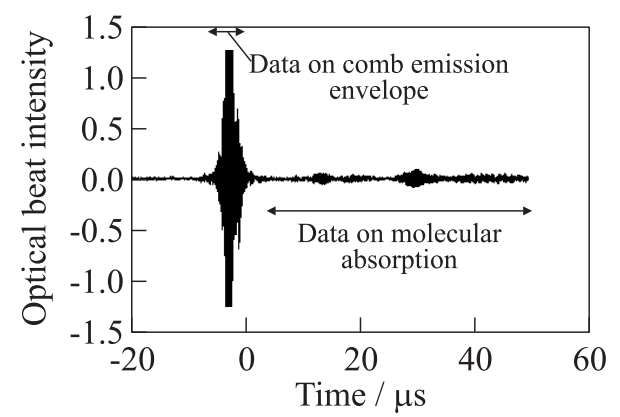

(a)

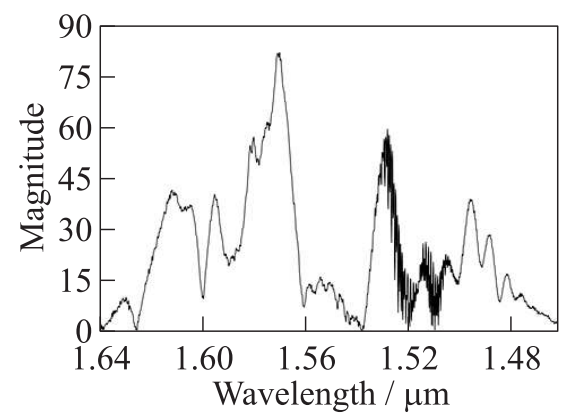

(b)

Figure 6 Dual comb Fourier spectrum: (a) typical interferogram obtained for the optical beating between the two frequency combs; and $(b)$ inverse Fourier transform of interferogram exhibiting $\mathrm{C}_{2} \mathrm{H}_{2}$ absorption lines 
spectrum of $\mathrm{C}_{2} \mathrm{H}_{2}$ depicted in Fig. $6 b$. Processing is still underway to derive quantitative values of the concentration of this specie.

New difficulties like coherence maintenance of the comb propagation in a chemically reactive high-temperature gas which may bring wavelength dispersion, greater sensitivity to luminous background are encountered and should be solved in order to apply this new technique for quantitative and robust broad band measurements in chemically reacting gas flows. Stabilization of the comb structure through locking of the two critical parameters (repetition frequency $f_{r}$ and offset frequency $f_{o}$ ) are crucial to obtain exploitable Fourier interferograms of the optical beatings between the combs. And only well frequency referenced combs will enable the characterization of any wavelength dispersion in the turbulent media. A partial solution which avoids active stabilization on the lasers is through autoadaptive acquisition where the repetition frequency $f_{r}$ and offset frequency $f_{o}$ must be acquired through optical beatings with two single mode stabilized lasers [13]. This will certainly help to obtain exploitable Fourier interferograms but will not allow interpreting dispersion features in the probed media.

Another solution is through nonlinear optical conversion (frequency doubling or DFG) of the comb to another spectral window which eliminates the offset frequency $f_{o}$.

We will be implementing soon the DFG scheme to translate the near-infrared comb to the mid-infrared region where most of molecules for our applications $\left(\mathrm{CO}, \mathrm{NO}, \mathrm{C}_{x} \mathrm{H}_{y}\right.$, etc.) have their fundamental and, hence, more intense absorption lines.

\section{RECENT PROGRESS IN LASER INDUCED FLUORESCENCE IMAGING FOR COMBUSTION DIAGNOSTICS}

Laser induced fluorescence imaging of $\mathrm{OH}, \mathrm{CO}$, and $\mathrm{NO}$ is under development at ONERA at high repetition rate in the kilohertz regime. The new LIF system now operated appears as a very valuable tool to allow understanding of combustion mechanism observed in a real combustor with adequate temporal resolution.

Laser induced fluorescence on metal atoms is also being demonstrated as a perfect tool for situ diagnostics of aluminized solid propellants. Laser induced fluorescence on atomic iron (Fe-LIF) was recently demonstrated to allow efficient visualization of flow dynamics, and $\mathrm{Al}$ and $\mathrm{Bi}$ atoms are under study to further investigate the combustion of the propellant. 


\subsection{Two-Photon Laser Induced Fluorescence Imaging on Carbon Monoxide for Detection of Pollutant Emissions from Aircraft Engines}

The first step of this development action was to develop a numerical code that simulates excitation and fluorescence spectra of $\mathrm{CO}$ molecule. To validate this code, detailed spectroscopic measurements were carried out in an opticalaccessible gas cell in order to determine the photophysics of $\mathrm{CO}$ fluorescence under various conditions of temperature, pressure, and carrier gas.

The two-photon $B^{1} \Sigma^{+}\left(v^{\prime}=0 \leftarrow \leftarrow X^{1} \Sigma^{+}\left(v^{\prime \prime}=0\right)\right.$ excitation spectrum is characterized by a strong and narrow $Q$ branch having a maximum at $230.104 \mathrm{~nm}$ and very weak $S$ and $O$ branches (Fig. $7 a$ ). This feature can be explained by the overlap of the $Q$ branch lines because of the similar values of the rotational constants for the $B^{1} \Sigma^{+}\left(v^{\prime}=0\right)$ state $\left(B_{e}=1.9481 \mathrm{~cm}^{-1}\right)$ and the $X^{1} \Sigma^{+}\left(v^{\prime \prime}=0\right)$ state $\left(B_{e}=1.9225 \mathrm{~cm}^{-1}\right)$. Fluorescence spectrum in Fig. $7 b$ presents a maximum at $519 \mathrm{~nm}$ (band $B(0)-A(2)$ ). A comparison between simulated and experimental excitation and fluorescence spectra at $300 \mathrm{~K}$ and 1 bar is also presented in Fig. $7 b$. Both excitation spectrum of $Q$-branch and fluorescence spectrum are obtained in a mixture composed of $2.6 \% \mathrm{CO}$ diluted in $\mathrm{N}_{2} / \mathrm{O}_{2}$ (with respective molar fractions of $80 \%$ and $20 \%$ ). Good agreement between experiment and simulation is observed for both spectra. In particular, the shape and the width of the excitation spectrum are well captured by numeric simulations. In the fluorescence spectrum, the full width at half maximum of each band is equal to $1.4 \mathrm{~nm}$.

Figure 8 displays the excitation spectrum recorded in a flame with equivalence ratio equal to 1.16. The best fit of the experimental spectrum is obtained from simulations with a temperature of $1750 \pm 50 \mathrm{~K}$. This value is similar to

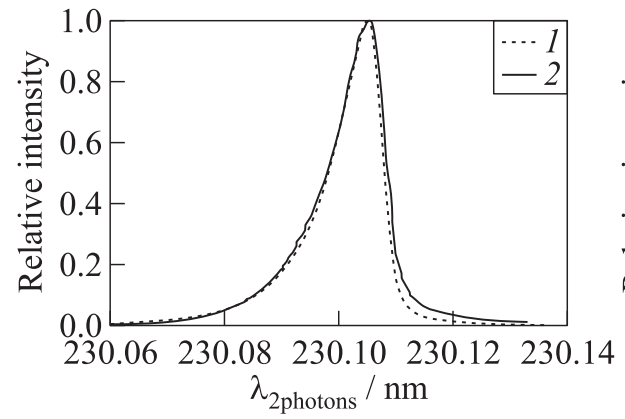

(a)

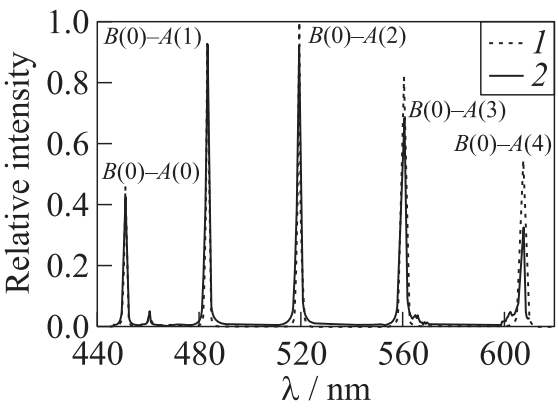

(b)

Figure 7 Comparison between simulation (1) and experiments (2) of excitation (a) and fluorescence $(b)$ spectra at $300 \mathrm{~K}$ and 1 bar 


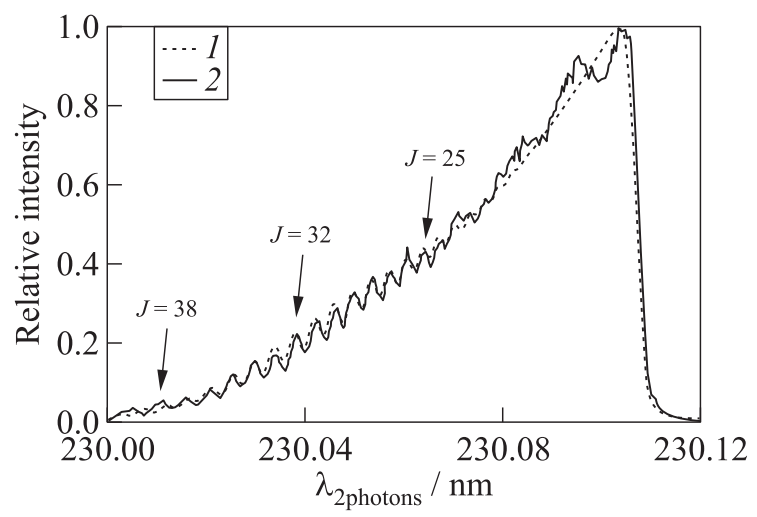

Figure 8 Comparison between simulation (1) and experiments (2) of integrated fluorescence in flame at 1 bar

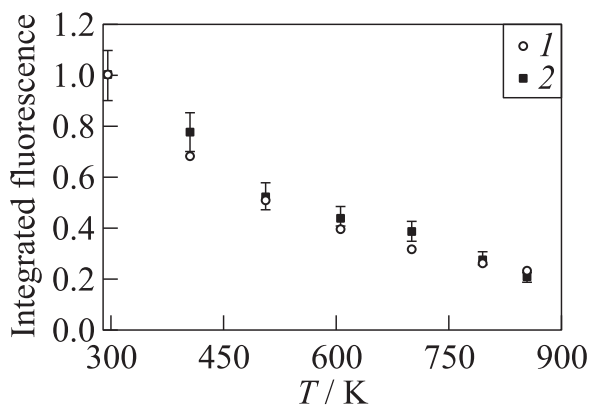

(a)

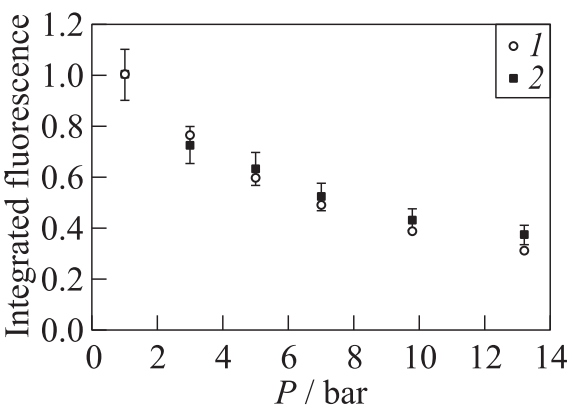

(b)

Figure 9 Comparison between simulation (1) and experiments (2) of integrated fluorescence as a function of temperature $(P=1 \mathrm{bar})(a)$ and pressure $(T=300 \mathrm{~K})(b)$

that predicted by an existing combustion code (PREMIX) at ONERA. At that temperature, rotational structure is resolved for levels with $J>25$. Indeed, increasing temperature induces a broadening of the $Q$-branch line shape and leads to the apparition of the rotational lines because collisional width decreases with temperature. Comparison between simulations and experiments is in good agreement. In particular, the amplitude of the spectral lines and the distance between each line is well reproduced in the simulations. The CO-LIF code is, therefore, validated for temperatures in the range 300-1750 K.

Variations of the integrated fluorescence with temperature at 1 bar and pressure at $300 \mathrm{~K}$ are illustrated in Fig. 9. Integrated fluorescence of the $B(0)-A(1)$ band is normalized by the peak signal at $300 \mathrm{~K}$ and 1 bar. Good agreement is 
observed between experiments and simulations. The CO-LIF code is, therefore, validated for pressures in the range 1-13 bars.

It has been noted that temperature and pressure induce a broadening of the $Q$-branch line shape. Additionally, unlike temperature, pressure induces a significant spectral shift on the excitation spectra. Indeed, increasing pressure from 1 to 10 bar shifts the $Q$-branch by $7 \mathrm{pm}$ towards the longest wavelengths. In the future, we will develop the CO-PLIF (planar LIF) imaging technique in a premixed lab-scale burner operating with methane fuel in order to determine the spatial distribution of $\mathrm{CO}$ molecules in a flame.

\subsection{Laser Induced Fluorescence Imaging Measurements on Iron Atoms in Solid Propellant Flames}

The goal of the present work is to develop the PLIF on atomic iron (Fe-PLIF) technique for in situ investigation of solid propellant combustion. Performing optical diagnostics in high-pressure solid propellant combustion remains a challenge, especially in actual conditions. Indeed, in industrial propellants, especially those based on ammonium perchlorate (AP) and a polybutadiene binder (e.g., hydroxyl-terminated polybutadiene, HTPB), combustion process is associated to ultrahigh luminosity of the flame due to high temperature and large continuous emission background which intensity increases with pressure. Although such high-pressure, high-luminosity, heavily particles laden environment of solid propellant combustion makes optical measurements difficult, these are required in order to better understand combustion phenomena and to provide data for the validation of modeling and numerical simulations.

The LIF technique has been pioneeringly applied by Edwards et al. [14] to investigate the flames of a monopropellant (HMX) and an AP/HTPB propellant at elevated pressures (up to $3.5 \mathrm{MPa}$ ). The authors pointed out:

(1) the difficulty to extract a proper LIF signal from the intense laser scattering by condensed particles in the propellant flame and from the continuous background emission (in the case of AP-based composite propellants);

(2) large laser beam attenuation and fluorescence trapping by the dense medium of high-pressure flames; and

(3) the challenge to account for the pressure-dependent quenching of LIF signal originating from molecular radicals $(\mathrm{OH}, \mathrm{CN}, \mathrm{CH}$, etc.) in order to derive quantitative results.

Later on, LIF technique has been extended to PLIF in order to obtain single-shot information over a flow area of a few cubic centimeters $[15,16]$. More recently, high-speed OH-PLIF operating at $5 \mathrm{kHz}$ was used by Hedman et al. [17] to 
investigate combustion process of bimodal $\mathrm{AP} /$ binder propellants at pressures between 0.1 and $0.64 \mathrm{MPa}$. The $\mathrm{OH}$ images were recorded close to the surface of the burning propellant in order to follow the flame dynamics, flame height, regression velocity of the propellant surface, and ejection of AP particles from the burning propellant.

Ballistic additives containing iron atoms such as ferrocenic-type catalysts are commonly incorporated inside the solid propellant formulations. The idea is to probe the iron atoms evaporated in the flame by LIF for the visualization of the combustion flowfield when hot gases are generated by the propellant combustion and when turbulent structures are developing above the propellant surface. For this purpose, the potential of iron atoms for the characterization of the hot gases flow, in particular, in the shear/vortices regions is investigated by the LIF technique which provides high spatial (micrometer) and temporal (nanosecond) resolution, good chemical selectivity, and an expected better signal-to-noise ratio than the above-mentioned molecular combustion species.

Laser excitation at $248 \mathrm{~nm}$ generated by a $\mathrm{KrF}$ excimer laser is used together with a detection of the fluorescence signal around $300 \mathrm{~nm}$. The experiments are performed for AP-based composite propellants doped with iron-containing catalysts under different flow conditions. The propellant samples are placed at the center of the combustor and are ignited by means of a pulsed $\mathrm{CO}_{2}$ laser. The laser for iron atoms probing is a $\mathrm{KrF}$ excimer laser ( $5 \mathrm{~ns}, 5 \mathrm{~mJ}$ pulses, $0.35 \mathrm{~nm}$ bandwidth, 25-kilohertz repetition rate). The central laser wavelength matches the strongest absorption line of atomic iron at 248.327 corresponding to the electronic transition $x^{5} \mathrm{~F}_{5}^{0}-a^{5} \mathrm{D}_{4}$ originating from the ground state [18]. The laser beam is transformed into a collimated sheet using a combination of cylindrical and spherical lenses before entering the combustion chamber equipped with ultraviolet (UV) fused silica windows (Fig. 10). The sheet thickness is $2.5 \mathrm{~mm}$ corresponding to half of the propellant sample thickness. Fluorescence is detected at $90^{\circ}$ from the laser axis by means of an intensified charge-coupled device (ICCD) (16 bit, $1024 \times 1024$ pixels, and 3-hertz framing rate). The temporal gate is set to $50 \mathrm{~ns}$. The camera is equipped with an UV objective which provides a $32 \times 32$-millimeter image (spatial resolution of $31 \mu \mathrm{m} / \mathrm{pixel}$ ) and with a band-pass filter to pass the fluorescence from iron atoms with high transmission (at $300 \mathrm{~nm}$ ) in a narrow spectral range (10-nanometer bandwidth) together with an additional filter in order to totally reject laser scattering by particles and smokes.

As depicted in Fig. 10, air flow is added to induce turbulent structures and one sample is doped with iron-based catalysts, while the other is not. The heights of the samples are different to create a step which results in a shear that leads to the development of vortices in the flow.

Figure 11 illustrates the single-shot Fe-PLIF images recorded above the surfaces of the two burning AP/HTPB propellants (with and without iron) at $0.3 \mathrm{MPa}$. The sample at the left-hand side contains approximately $1 \%$ (wt.) 


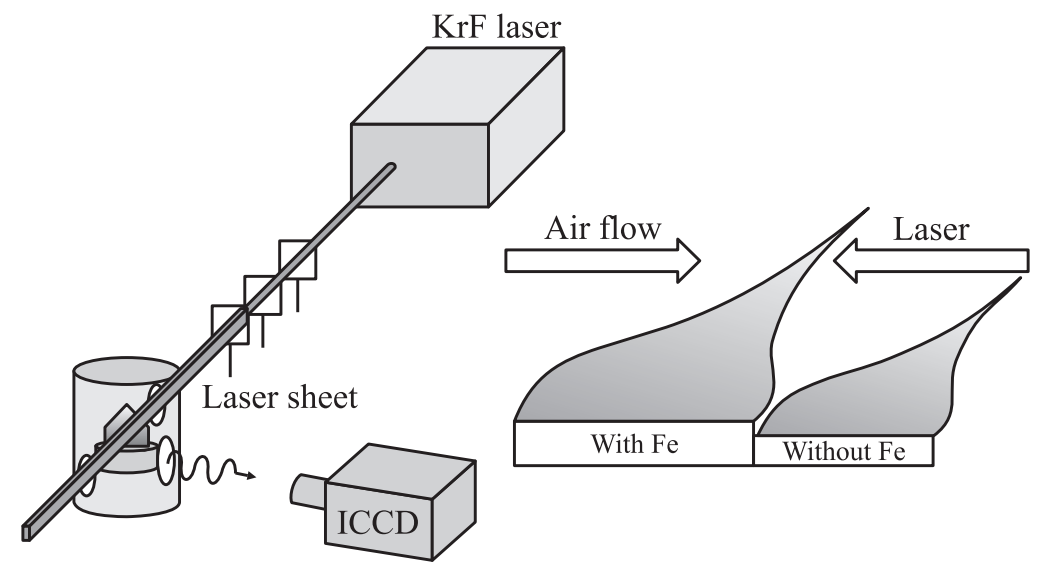

Figure 10 Sketch of the PLIF setup for propellant flames visualisation using Fe atoms as tracer

of iron. The LIF signal from iron atoms is observed only in the hot plume of the iron-containing propellant which asserts that the signal is free from interferences and purely due to the selected emission transition of Fe atom. It proves that the chosen laser excitation / fluorescence detection wavelengths couple is iron selective despite the numerous species produced in such flames $(\mathrm{HCl}$, iron oxides, and iron chlorides in addition to the conventional combustion molecules).

The frontier region between the two flames (with and without iron) is clearly marked by the interface between the region of high level of signal (above the iron-containing propellant) and the dark region where signal is zero (above the Fe-free propellant). The signal reaches very high levels and remains constant at larger distance with a homogeneous radial distribution in the flame. A steep gradient of signal is observed over $1.5 \mathrm{~mm}$ just above the surface propellant (see Fig. 11a) owing to the rapid rise of the temperature from the propellant surface $(\sim 700-900 \mathrm{~K})$ and the flame temperature $(>2000 \mathrm{~K})$ over a small height (few hundreds of micrometer). One observes clearly large-scale flow structures corresponding to the gradual disappearance of the initial laminar boundary layer between the cold air flow and the hot gases generated by the flame. Indeed, shear stress between the two jets tends to destroy the laminar boundary layer and generates coherent structures that trigger the instability of the shear layer.

As a result, a typical turbulent structure called mushroom-like vortex (with two counterrotating vortices) forms when the instability of the shear layer is fully developed and is observed on the right-hand side of the image. Iron atoms accumulation in the "mushroom head" is observed, although fluorescence intensity is reduced by a factor of two compared to the signal above the propellant, 


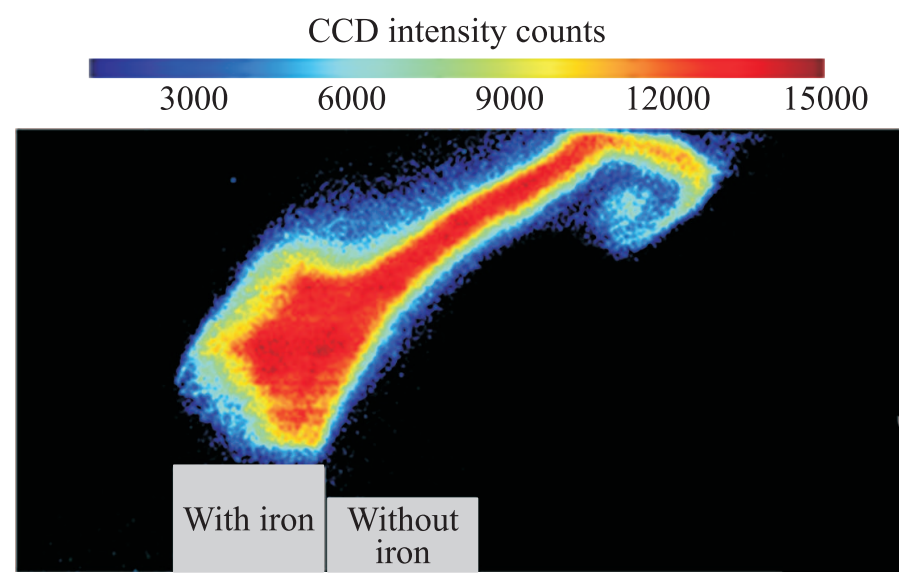

(a)

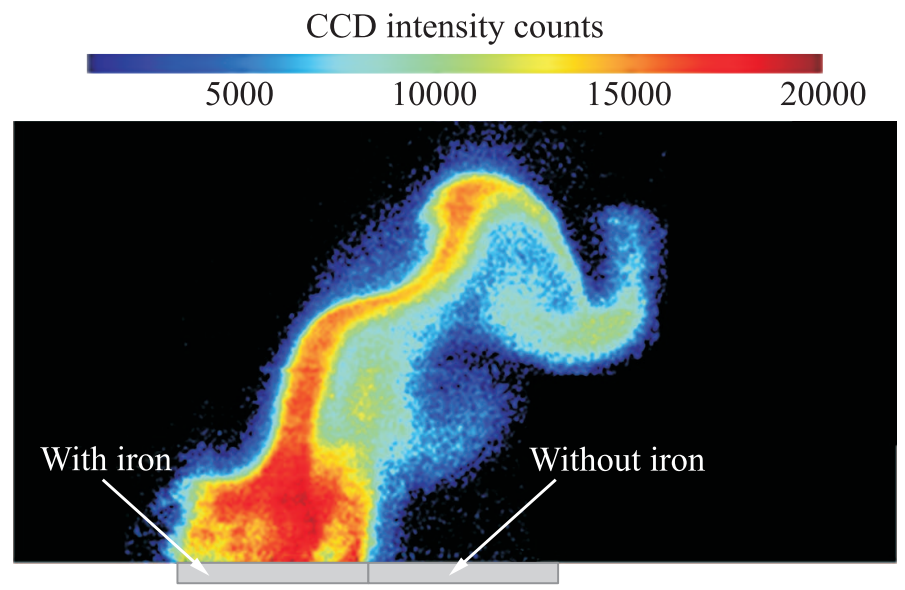

(b)

Figure 11 Single-shot Fe-PLIF images obtained above the surfaces of burning $\mathrm{AP} / \mathrm{HTPB}$ propellants at $0.3 \mathrm{MPa}$ : (a) $1.5 \mathrm{~s}$ after ignition, and (b) end of combustion sequence. Size of the image: $30 \times 16 \mathrm{~mm}$

possibly due to air dilution. Such image shows that iron atoms are present at the periphery of the large-scale structures. It is noted that the short gate (50 ns) of the ICCD camera enables to freeze the flow pattern and track turbulent flow structures during the combustion process of iron-doped solid propellants. However, the limited acquisition rate of the camera does not allow to resolve the temporal evolution of these turbulent structures. 
This result demonstrates the potential of Fe-PLIF to visualize hot gas flow fields in solid propellant combustion with a large signal-to-noise ratio and suggests that the technique may be applied at higher pressures (beyond $2 \mathrm{MPa}$ ), knowing that high levels of signal and also persistent over a large distance are already obtained at $1 \mathrm{MPa}$ (not shown in the present article).

To go further, Fe-PLIF technique at a medium repetition rate of $300 \mathrm{~Hz}$ will soon be applied to a combustor representative of an actual rocket motor (pressure up to $4 \mathrm{MPa}$, large dimensions of solid propellants, possibly opticallythick medium). In the future, it will be also necessary to increase the repetition rate of the technique to kilohertz values in order to better follow the rapid flame and flow dynamics. For example, velocity information could be extracted from successive high-speed Fe-PLIF images by means of optical flow methods. Such information would be extremely useful for the validation of Large Eddy Simulations (LES) in solid propellant combustion.

\subsection{Towards the High-Speed Laser Induced Fluorescence Imaging}

High-speed OH-PLIF imaging for the characterization of the turbulent and reacting flows is currently under development in our laboratory. Up to now, nanosecond pulsed dye lasers with a repetition rate of $10 \mathrm{~Hz}$ are used and are coupled with ICCD camera whose framing rate is limited to $3 \mathrm{~Hz}$. With the aid of the novel high-speed nanosecond dye lasers and high framing intensified cameras, the PLIF technique at $1-10$-kilohertz regime is now available. This high temporal resolution offers the possibility to visualize the turbulent combustion events that occur on the time scale of milliseconds. For instance, Boxx et al. have applied 5-kilohertz OH-PLIF on lifted jet flames and swirl stabilized flames [19] and Hedman et al. have performed 5-kilohertz OH-PLIF imaging close to the burning surface of AP/HTPB propellants [20]. The latters were able to record a sequence of several images of the combustion of a coarse AP particle at $1 \mathrm{~atm}$ over a time scale of $300 \mathrm{~ms}$.

Our group has now been equipped with a 10-kilohertz tunable dye laser (Sirah Credo) which delivers $400 \mu \mathrm{J}$ per pulse at $283 \mathrm{~nm}$ (or $4 \mathrm{~W}$ of average power) with 5-nanosecond pulse duration. The dye laser is pumped by a diode-pumped Nd:YAG laser which is frequency doubled (532 nm) delivering almost 100-watt pulses at $10 \mathrm{kHz}$ (Edgewave, Innoslab). Currently, the laser wavelength is tuned to the rotational line $Q(6)$ of the $A^{2} \Sigma(1)-X^{2} \Pi(0)$ of $\mathrm{OH}$ at $282.750 \mathrm{~nm}$ (in vacuum). The laser line width is $0.12 \mathrm{~cm}^{-1}$ (full width at half maximum (FWHM)). The PLIF images are recorded by using a high speed camera $(1024 \times 1024$, 12 bit, CMOS, HighSpeedStar, Lavision) coupled with a gated UV intensifier (HighSpeedIRO, Lavision).

High-speed OH-PLIF will be first applied in a rocket exhaust plume in the frame of an internal research program dedicated to the fundamental problems 
related to plume flow fields of solid or liquid rocket engines. The high-speed $\mathrm{OH}-\mathrm{PLIF}$ images will be recorded in the supersonic exhaust plume downstream the nozzle. This investigation will focus on the regions of the postcombustion and of the mixture layer located in the jet periphery with the aim of tracking the turbulences of the reactive jet. In that study, the representative combustor will be fed by gaseous methane and oxygen (MASCOTTE facility at ONERAPalaiseau). The operation pressure will be set at 20 bar and the mixture ratio at 1.5. The OH-PLIF images will be compared to the LES of the turbulent mixing layer between the plume and the external environment.

An innovative research work with the aim of probing atomic aluminum vapors in solid propellant flames using high-speed LIF is currently conducted in our laboratory. Few experimental data of in situ combustion of aluminized solid propellant in realistic conditions exist owing to the harsh environmental conditions (high-temperature, high-pressure, high-luminous, particles laden flames, highly turbulent and short combustion duration less than $1 \mathrm{~s}$ ) make optical measurements very difficult. New strategies for optical diagnostics in solid propellant flames are in progress and are based on gaseous aluminum and iron atoms as fluorescent markers. The above-mentioned high repetition rate laser will be used to probe aluminum atoms in simplified test experiments in order to develop our strategy for high-speed Al-PLIF.

\section{COHERENT ANTI-STOKES RAMAN SPECTROSCOPY WITH FEMTO/PICOSECOND LASERS}

ONERA has been a pioneer in the coherent anti-Stokes Raman spectroscopy (CARS) technique applied to combustion diagnostics [21]. The development effort is now dedicated to a hybrid femto-picosecond CARS setup to retrieve information such as temperature or chemical composition. The aim is to probe fast temporal dynamics in combustion with high repetition rate (kilohertz), as recently demonstrated by several authors $[22,23]$.

\subsection{Hybrid Pico/Femtosecond-Coherent Anti-Stokes Raman Spectroscopy Setup}

The CARS is a four-wave mixing process that relies on 2-photon (pump and Stokes) excitation of molecular coherences between pairs of ro-vibrational states and their probing by a third photon called Probe. The generated anti-Stokes signal provides molecular specific information from the medium, giving access to 
quantities such as relative concentration of the major species or rotational and vibrational temperature of the gas.

Coherent anti-Stokes Raman spectroscopy has been historically developed in the nanosecond regime, using narrowband pulsed lasers to excite the molecular gas. When the Pump-Stokes detuning matches a Raman active vibration, the anti-Stokes signal is maximized. Therefore, scanning the detuning of the Stokes and the Pump frequencies was first used to retrieve the whole Raman ro-vibrational spectrum. Later on, broadband Stokes (or Pump) laser pulse has been demonstrated to generate the whole anti-Stokes spectrum in a single laser shot. The signal is further analyzed by a spectrometer to follow the temporal evolution of the medium parameters from shot to shot.

The latest efforts on CARS diagnostics were focused on the increase of the CARS measurements repetition rate, so that short temporal dynamic phenomena could be probed. Indeed, nanosecond CARS was limited to $10-30 \mathrm{~Hz}$ due to the high energy pulse required for single shot measurements, although multikilohertz measurements are required to resolve turbulent media evolution. A solution appeared recently, since ultrafast femtosecond lasers now permit to reach high enough peak powers to get strong enough single shot CARS signal at high repetition rate. Thus, kilohertz CARS thermometry was demonstrated at first by using the Chirped Probe Pulse technique [22], or later on using the hybrid pico-femtosecond regime [23]. The latter takes advantage of the two temporal regimes in order to provide:

(1) a high peak power broadband femtosecond Pump/Stokes excitation that allows single shot multiplex measurement; and

(2) a picosecond narrowband Probe from which the anti-Stokes spectrum is generated with high enough spectral resolution, thus overcoming previous limitation of the femtosecond regime.

An experimental hybrid pico/femtosecond-CARS setup has been built at ONERA for combustion diagnostic purposes. As sketched in Fig. 12, the design uses a single Yb-laser source at $1030 \mathrm{~nm}$ to provide the Pump, Stokes, and Probe beams. An optical parametric amplification (OPA) with a doubling stage is used to generate the tunable broadband Stokes, while the 515-nanometer Pump comes from the second harmonic generation (SHG). The narrowband picosecond Probe is generated using an ultranarrow filter to provide a good resolution on the spectroscopy $\left(1 \mathrm{~cm}^{-1}\right)$. The beams are synchronized using delay lines and focused in the medium in a folded BOXCARS geometry. The anti-Stokes signal is filtered with dichroics and analyzed using a spectrometer coupled to an electron-multiplying CCD camera.

As illustrated in Fig. 13, the laser line generation has been designed in a very robust, compact, and mobile setup in order to be brought as close as possible to the combustion facilities. 


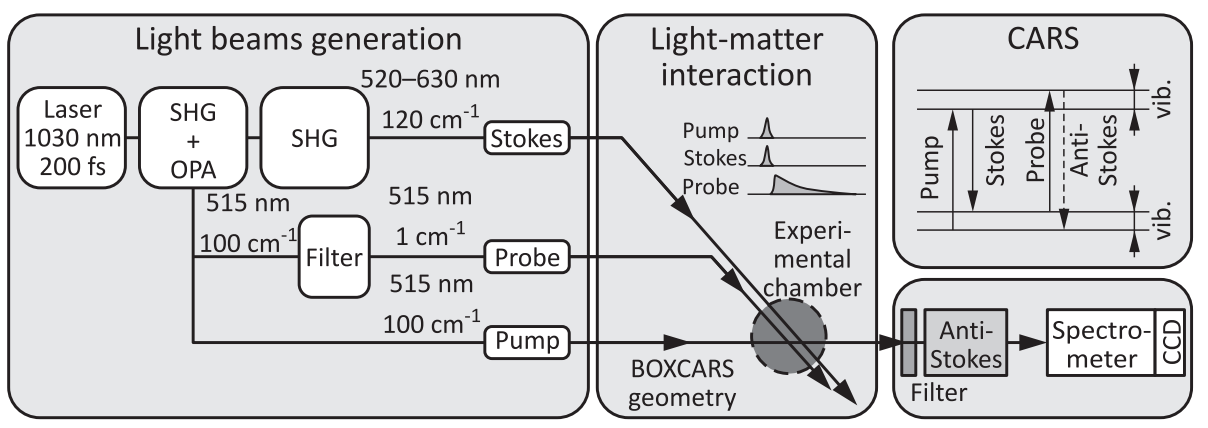

Figure 12 Experimental scheme of the pico/femtosecond-CARS setup at ONERA. Over each beam are listed the mean wavelength (in $\mathrm{nm}$ ) and the spectral width $\left(\mathrm{FWHM}\right.$, in $\mathrm{cm}^{-1}$ )

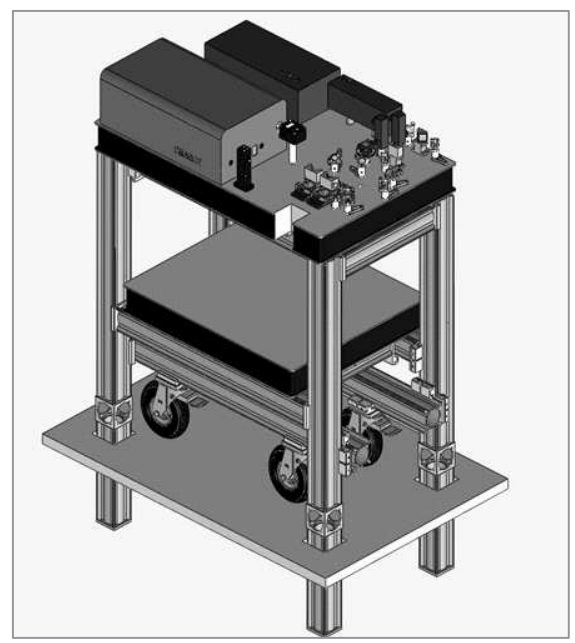

Figure 13 Three-dimensional view of the experimental CARS setup at ONERA

\subsection{Nonresonant Contribution Suppression in Pico/Femtosecond-CARS Spectra}

This experimental setup has been used in order to record $\mathrm{N}_{2}$ CARS spectra in a stoichiometric $\mathrm{CH}_{4}$ /air flame. The high-temperature $(\sim 2000 \mathrm{~K})$ spectra plotted in Fig. 14 exhibit typical vibrational bands, making it suitable for thermometry measurements. In Fig. 14a, the delay between the Pump-Stokes excitation and the Probe pulse has been set to zero; so, the three pulses are 


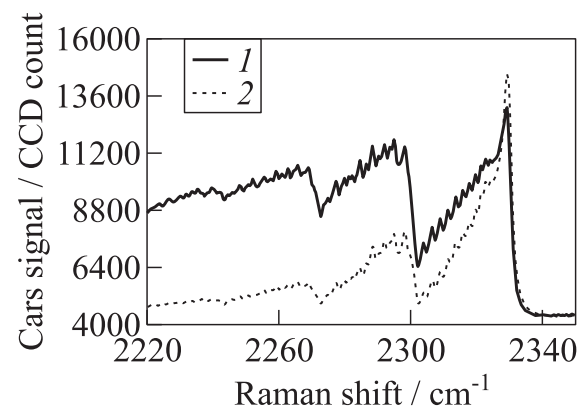

(a)

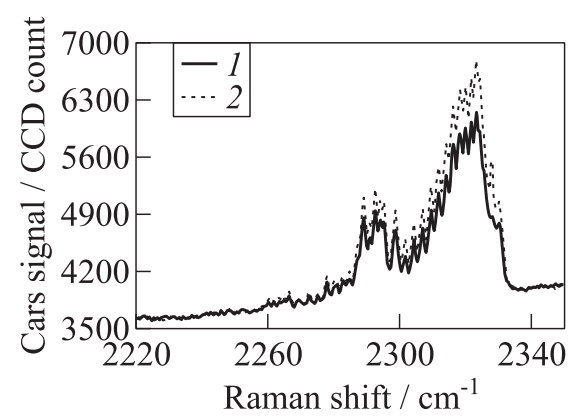

(b)

Figure $14 \mathrm{~N}_{2}$ CARS spectra using a parallel (1) or crossed polarized (2) Probe pulse: $(a)$ at zero Pump/Probe delay; and $(b)$ at 10-picosecond Pump/Probe delay

temporally overlapping. In this case, CARS signal appears superimposed over an intense nonresonant background. Indeed, Probe polarization was set either parallel or crossed as regard to Pump and Stokes vertical polarizations and the spectra show a clear difference on the mean nonresonant contribution with respective high or low distortion of the resonant features. In Fig. 14b, the Probe pulse has been decoupled from the excitation by introducing a 10-picosecond delay. The spectrum is modified, some coherences being selectively enhanced, and vibrational bands can be identified. Moreover, as expected [24], the change from parallel to crossed polarization scheme does not produce a significant change in the measured spectra. Indeed, nonresonant contribution lifetime being of the order of a few $100 \mathrm{fs}$, a delayed probe is effectively removing this harmful contribution. Thus, hybrid pico/femtosecond regime could be efficiently used to record $\mathrm{N}_{2}$ ro-vibrational spectra and to remove nonresonant contribution, which was one of the major limitations of nanosecond CARS.

\section{CONCLUDING REMARKS}

This paper presented some of the recent development work carried out at ONERA on spectroscopic techniques for aerodynamic and combustion flows. Most of the techniques benefit from recent advances in lasers, particularly, from now available features like high repetition rates, short duration (pico/ femtosecond), and frequency comb emission. The results obtained up to now are encouraging and further developments are under way on the abovementioned techniques as well as on others like laser induced breakdown for high altitude relight of aircraft engines. More consolidated data will be published soon. 


\section{ACKNOWLEDGMENTS}

The CARS, Frequency Comb, and LIF setups have been partially funded by the PARIS region in France (ASTRE and SESAME frameworks).

\section{REFERENCES}

1. Mohamed, A.K., and M. Lefebvre. 2009. Laser absorption spectroscopy to probe chemically reacting flows. AerospaceLab J. 1. Al01-13. 12 p.

2. Keilmann, F., C. Gohle, and R. Holzwarth. 2004. Time-domain mid-infrared frequency-comb spectrometer. Opt. Lett. 29:1542-1544.

3. Adler, F., M. J. Thorpe, K. C. Cossel, and J. Ye. 2010. Cavity-enhanced direct frequency comb spectroscopy: Technology and applications. Annu. Rev. Anal. Chem. 3(1):175-205.

4. Ye, J., and S. T. Cundiff, eds. 2005. Femtosecond optical frequency comb: Principle, operation and applications. Springer. $374 \mathrm{p}$.

5. Hall, J. L. 2006. Nobel lecture: Defining and measuring optical frequencies. Rev. Mod. Phys. 78:1279.

6. Udem, T., R. Holzwarth, and T.W. Hänsch. 2002. Optical frequency metrology. Nature 416:233-237.

7. Shirasaki, M. 1996. Large angular dispersion by a virtually imaged phased array and its application to a wavelength demultiplexer. Opt. Lett. 21(5):366-368.

8. Diddams, S. A., L. Hollberg, and V. Mbele. 2007. Molecular fingepriniting with the resolved modes of a femtosecond laser frequency comb. Nature 445:627-630.

9. Gohle, C., B. Stein, A. Schliesser, Th. Udem, and T. W. Hänsch. 2007. Frequency comb Vernier spectroscopy for broadband, high-resolution, high-sensitivity absorption and dispersion spectra. Phys. Rev. Lett. 99:263902.

10. Schiller, S. 2002. Spectrometry with frequency combs. Opt. Lett. 27:766-768.

11. Coddington, I., W. Swann, and N. Newbury. 2008. Coherent multiheterodyne spectroscopy using stabilized optical frequency combs. Phys. Rev. Lett. 100:013902.

12. Mandon, J., G. Guelachvili, and N. Picqué. 2009. Fourier transform spectroscopy with a laser frequency comb. Nat. Photonics 3:99-102.

13. Ideguchi, T., A. Poisson, G. Guelachvili, N. Picqué, and T. W. Hänsch. 2014. Adaptive real-time dual-comb spectroscopy. Nat. Commun. 5:3375. doi: 10.1038/ncomms4375.

14. Edwards, T., D. P. Weaver, and D. H. Campbell. 1987. Laser-induced fluorescence in high pressure solid propellant flames. Appl. Opt. 26:3496-3509.

15. Parr, T., and D. Hanson-Parr. 2000. Optical diagnostics of solid-propellant flame structures. Solid propellant chemistry, combustion, and motor interior ballistics. Eds. V. Yang, T. Brill, and W.Z. Ren. Progress in astronautics and aeronautics ser. Reston, VA: AIAA. 185:381-411. Ch. 2.5. 
16. Parr, T., and D. Hanson-Parr. 2004. Cyclotetramethylene tetranitamine/glycidyl azide plymer/butanetriol trinitrate propellant flame structure. Combust. Flame 137:38-49.

17. Hedman, T. D., K. Y. Cho, M. Pfeil, A. Satija, H. Mongia, L. Groven, S. F. Son, and R. P. Lucht. 2012. High speed PLIF applied to multiphase combustion. Spring Technical Meeting of the Central States Section of the Combustion Institute.

18. Fuhr, J.R., and W.L. Wiese. 2006. A critical compilation of atomic transition probabilities for neutral and singly ionized iron. J. Phys. Chem. Ref. Data 35:16691753.

19. Boxx, I., M. Stöhr, C. Carter, and W. Meir. 2009. Temporally resolved planar measurements of transient phenomena in a partially pre-mixed swirl flame in a gas turbine model combustor. Appl. Phys. B 95(1):23-29.

20. Hedman, T.D., K. Y. Cho, A. Satja, L. J. Groven, R. P. Lucht, and S. F. Son. 2012. Experimental observation of the flame structure of a bimodal ammonium perchlorate composite propellant using $5 \mathrm{kHz}$ PLIF. Combust. Flame 159(1):427437.

21. Druet, S. A. J., and J. P. Taran. 1981. CARS spectroscopy. Prog. Quant. Electron. 7:1-72.

22. Roy, S., W.D. Kulatilaka, D. R. Richardson, R. P. Lucht, and J. R. Gord. 2009. Gas-phase single-shot thermometry at $1 \mathrm{kHz}$ using fs-CARS spectroscopy. Opt. Lett. 34:3857.

23. Miller, J. D., M. N. Slipchenko, T. R. Meyer, H. U. Stauffer, and J. R. Gord. 2010. Hybrid femtosecond/picosecond coherent anti-Stokes Raman scattering for highspeed gas-phase thermometry. Opt. Lett. 35:2430.

24. Zinth, W. 1980. Transcient cohenrent Raman scattering in the time and frequency domain. Opt. Commun. 34:479. 